\title{
EL MANEJO DE PROYECTOS ECOTURÍSTICOS EN EL MUNICIPIO DE SAN CRISTÓBAL DE LAS CASAS: ¿SIGUEN LA NORMA OFICIAL MEXICANA 133?
}

\author{
JoSÉ GERARDO DOMÍNGUEZ-VERA \\ gerardodominguez020695@gmail.com \\ LORENA RUIZ-MONTOYA \\ Iruiz@ecosur.mx \\ El Colegio de la Frontera Sur
}

Evaluar el manejo de proyectos ecoturísticos conforme a normas preestablecidas es importante para asegurar resultados positivos. Teniendo presente lo anterior, el objetivo de este trabajo consiste en analizar la situación actual del servicio de ecoturismo en cuatro proyectos en el municipio de San Cristóbal de las Casas y determinar el grado de aplicación de los criterios de sustentabilidad que marca la Norma Oficial 133 (NMX-AA-133-SCFI-2013). Mediante observaciones y entrevistas se puntuaron los criterios de la referida Norma. Dos de los sitios tuvieron puntajes cercanos al mínimo necesario para considerarse con prácticas apegadas al lineamiento $y$, por lo tanto, con manejo sustentable.

Palabras clave: ecoturismo, normas, planeación, conservación y áreas naturales.

\section{DOES THE MANAGEMENT OF ECOTOURISM PROJECTS IN THE MUNICIPALITY OF SAN CRISTÓBAL DE LAS CASAS FOLLOW THE OFFICIAL MEXICAN STANDARD 133?}

Evaluating the management of ecotourism projects according to pre-established norms is important to ensure positive results. Bearing this in mind, the objective of this work is to analyze the current situation of the ecotourism service in four projects in the municipality of San Cristóbal de las Casas and determine the degree of application of the sustainability criteria established by Official Standard 133 (NMX-AA-133-SCFI-2013). Through observations and interviews, the criteria of the referred Standard were scored. Two of the sites had scores close to the minimum necessary to be considered with practices adhering to the guideline and, therefore, with sustainable management.

Keywords: ecotourism, standards, conservation, planning, natural areas.

Fecha de recepción: 10 de marzo de 2021 Fecha de aceptación: 15 de mayo de 2021

CÓMO CITAR: Domínguez-Vera, J. y Ruiz-Montoya, L. (2021). El manejo de proyectos ecoturísticos en el municipio de San Cristóbal de las Casas: ¿siguen la norma oficial mexicana 133? Dimensiones Turísticas, 5(9), 59-80. https://doi.org/10.47557/DYFV8352 


\section{Introducción}

n México se ha decretado la norma NMX-AA-133-SCFI-2013, conocida como Norma 133, con el objetivo de establecer criterios de evaluación que garanticen el desempeño sustentable del ecoturismo (turismo ecológico o de bajo impacto) en el país, de acuerdo con la Secretaría de Medio Ambiente y Recursos Naturales (Semarnat, 2018). De acuerdo con información de la referida Secretaría, en el estado de Chiapas se encuentran seis destinos certificados bajo dicha norma: Ara Macao, Las Guacamayas, Guías de Siyaj Chan, Servicio Turístico Lacandones, Campamento Top Che, Campamento Ecoturístico Naha y Campamento Ya Toch Barum.

Sin embargo, existen iniciativas de ecoturismo en el municipio de San Cristóbal de las Casas, Chiapas, de las que se desconoce si cumplen con los criterios de la Norma 133. Entre ellas destacan los Parques ecoturísticos Arcotete y Grutas de Rancho Nuevo (Rancho Nuevo). De igual forma, se localizan áreas naturales protegidas privadas como Moxviquil y públicas como el Parque Educativo San José, que desarrollan actividades turísticas consideradas respetuosas del medio ambiente (ver figura 1).

Figura 1. Ubicación de las áreas de estudio
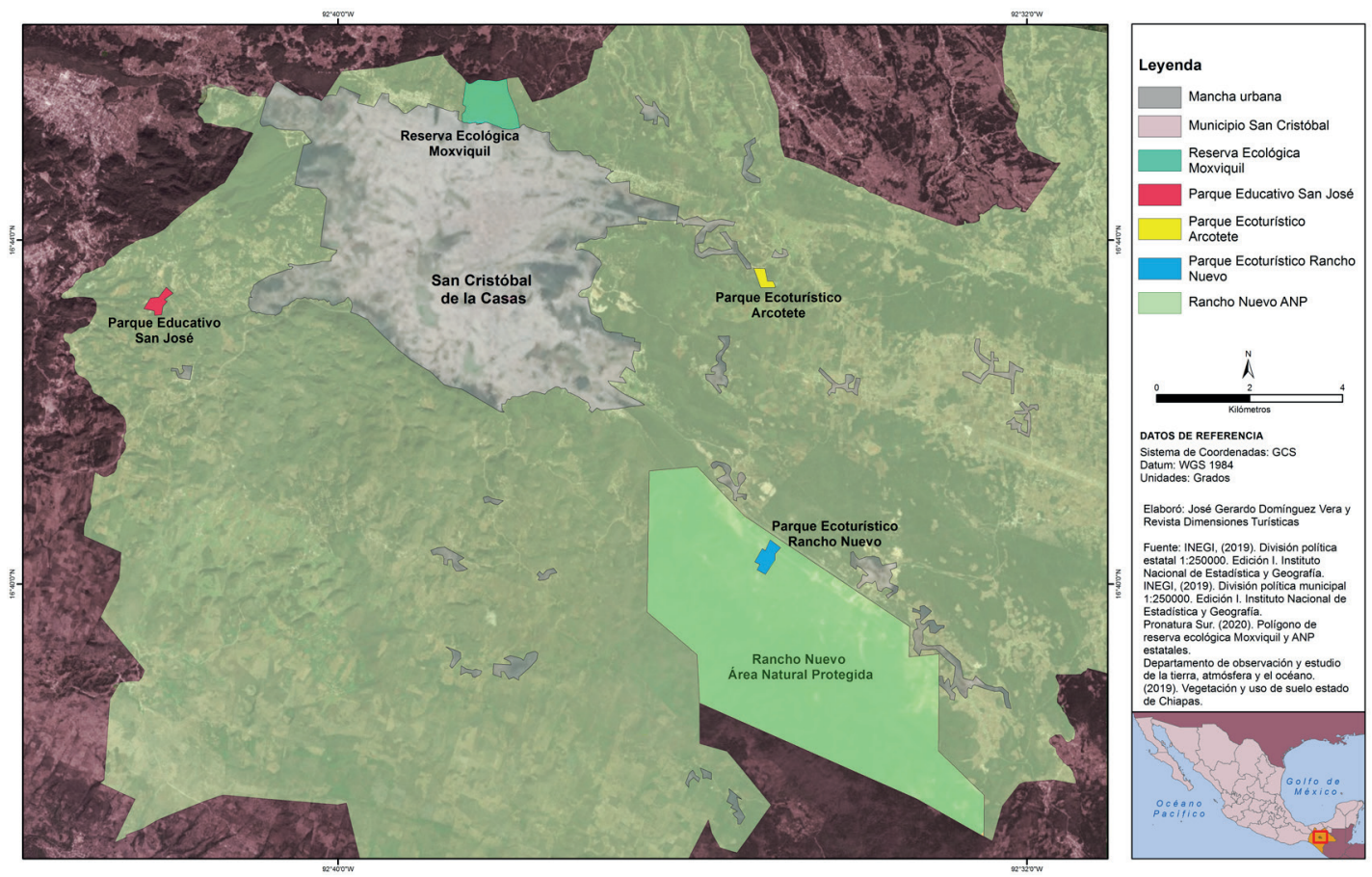

Fuente: Ver interior figura 1. 
El Parque Rancho Nuevo es un espacio de aproximadamente 20 hectáreas de bosque de pinos, perteneciente a la zona sujeta a conservación ecológica del mismo nombre (Instituto Nacional de Estadística y Geografía, INEGI, 2017) propiedad de la Secretaría de la Defensa Nacional (Sedena) pero administrada por la Sociedad Cooperativa de Servicio Turístico (Henríquez, 2012).

El Parque Arcotete actualmente es gestionado por 136 socios indígenas tsotsiles del ejido Carmen Arcotete (observación de campo) y cuenta con una extensión de 25 hectáreas de bosque de pino-encino y ciprés (Montoya y Hernández, 2013; Picado et al., 2014). Moxviquil es un área destinada a la conservación de 97 hectáreas, con vegetación secundaria de encino (Romero-Águila, 2012) y es administrada por la Asociación Civil Pronatura Sur. El Parque Educativo San José, adscrito a la Secretaría de Medio Ambiente e Historia Natural (Semahn), es una reserva de 16 hectáreas de bosque de pino-encino que fue abierto al público en junio de 1995 (Luna, 2018).

Los estudios previos respecto al crecimiento y organización de los parques Arcotete y Rancho Nuevo indican que el ecoturismo ha sido beneficioso para las comunidades, visto desde la perspectiva económica (Montoya y Hernández, 2013; Picado et al., 2014). No obstante, hay vacíos de información con relación a la parte ambiental, la cual es necesaria para reconocer los alcances del ecoturismo respecto a la conservación de los recursos naturales. En contraste, los estudios de Moxviquil y Parque Educativo San José se han desarrollado desde la perspectiva biológica y ambiental (Chanona-Gómez et al., 2019; Moreno, 2014; Romero-Águila, 2012). En general, hacen falta investigaciones acerca del manejo integral que supone el turismo visto bajo el marco de la sostenibilidad.

El presente estudio se realizó con el fin de identificar cómo se maneja el ecoturismo en dos sitios administrados por ejidos y dos áreas naturales (una privada y otra de carácter estatal); en particular si el manejo se basa en los fundamentos y principios con los que se concibe dicha actividad en México, en este caso bajo la Norma 133. Los objetivos que guiaron esta investigación fueron: 1) analizar la situación actual del servicio en los cuatro proyectos antes mencionados y 2) determinar el grado de aplicación de los criterios de sustentabilidad que marca la Norma.

Teniendo presente su finalidad, además de la introducción y las conclusiones, este documento se organiza en cuatro apartados: primeramente, se incluye un marco de referencia para lograr una mejor comprehensión del ecoturismo y la importancia de la normativa mexicana para su gestión. Enseguida, se describe la metodología 
utilizada para alcanzar los objetivos (el eje fue la aplicación de los criterios establecidos en la Norma 133 en particular); posteriormente, se presentan los resultados obtenidos, para finalmente discute la conveniencia de hacer evaluaciones de los proyectos ecoturísticos utilizando dicha norma como guía.

\section{Marco de referencia: el ecoturismo y la importancia de las normas mexicanas}

El ecoturismo, entendido como una modalidad del sector económico de los servicios, es un tipo de turismo que ha sido objeto de múltiples acercamientos $y$, por ende, existe una diversidad de definiciones sobre su significado; no obstante, para los fines de este trabajo se optó por la propuesta de Björk (2000, pp.196-197), porque se considera reúne de manera sintética la noción que se puede desprender de la Norma 133, refiriéndolo como "una actividad en la que las autoridades, la industria del turismo, los turistas y la población local cooperan para que los turistas puedan viajar a áreas naturales o silvestres para admirar, estudiar y disfrutar de la naturaleza y la cultura de una manera que no explote el recurso, pero contribuya al desarrollo sostenible".

De lo anterior se desprende que la práctica de esta actividad simultáneamente contribuye a la conservación que simultáneamente contribuye a la conservación de los recursos naturales, al bienestar de las comunidades locales y al desarrollo económico a largo plazo (Das y Chatterjee, 2015; Holub, 2015). Uno de los principios fundamentales del ecoturismo es promover actividades de observación e interpretación de la naturaleza, fomentar y fortalecer la educación ambiental de la sociedad local y del turista (Piñar et al., 2012; TIES, 2015).

Asimismo, este turismo demanda una participación activa por parte de los locales en la planificación y desarrollo del ecoturismo dentro de su territorio (PNUMA y OMT, 2002) y la distribución de responsabilidades entre los actores involucrados en el cuidado, respeto y conservación de los elementos naturales y culturales (Björk, 2000; TIES, 2015).

Hipotéticamente, este tipo de turismo ejerce una menor presión sobre los recursos renovables y no renovables, pero al mismo tiempo demanda una mayor inversión en capacitación y en monitoreos de impactos (Bringas y Ojeda, 2000). Pretende evitar la transformación del paisaje natural y ser una fuente de ingresos económicos 
para las comunidades locales (TIES, 2015). A diferencia de otras modalidades, el turismo enmarcado bajo la filosofía de la sostenibilidad es multidisciplinario porque integra componentes sociales, ambientes y económicos al momento de su planeación y desarrollo.

Aun cuando sus enfoques y fines pueden cambiar para ajustarse al contexto natural y social de un sitio, es absolutamente necesario crear instrumentos de evaluación que permitan garantizar que los servicios sean seguros para el visitante, la sociedad local y para la conservación de especies silvestres que le dan la belleza escénica y la complejidad ecológica que se incluye en la promesa del servicio (Camacho-Ruiz et al., 2016).

Una de las estrategias que se ha implementado a nivel mundial para alcanzar los beneficios del ecoturismo es la creación de certificaciones (Holub, 2015). Honey y Rome (2001, p. 5) las definen como: "un procedimiento voluntario que evalúa, monitorea y da garantía por escrito de que un negocio, producto, proceso, servicio o sistema de gestión se ajusta a requisitos específicos. Otorga un logotipo o sello comercializable a aquellos que cumplen o superan los estándares básicos".

Así, las certificaciones ecoturísticas se otorgan cuando se verifica que el desarrollo de los proyectos contribuye a la conservación ambiental y el desarrollo social local (Cancino, 2016). También constituye una manera de mostrar a los prestadores de servicios ecoturísticos la importancia de realizar evaluaciones de los proyectos con instrumentos propios y con base en normas oficiales para mejorar sus servicios (Holub, 2015). Los beneficios de obtener una certificación mexicana incluyen tener una gestión en el uso de los elementos naturales, la competitividad y destacarse como empresas de desempeño sustentable (Semarnat, 2018).

En México, la Semarnat es la institución que ha contribuido de manera más activa en el diseño del marco legal y los procedimientos para regular estos servicios en el país (Guerrero, 2010). Para lo cual se apoya en la Ley General de Equilibrio Ecológico y Protección al Ambiente, la Ley General de Vida Silvestre, la Ley General para la Prevención y Gestión Integral de los Residuos, la Ley Federal de Responsabilidad Ambiental, la Ley Federal del Mar y la Ley General de Bienes Nacionales (Semarnat, 2017), así como la certificación bajo la ya citada Norma 133 que establece las especificaciones de sustentabilidad a las que un prestador de servicios puede acreditarse. 


\subsection{Impactos del ecoturismo}

El tema del ecoturismo ha sido objeto de un debate internacional en diversos campos del conocimiento (económico, social y ambiental) debido a la falta de coincidencia entre lo que se ha establecido en la teoría y lo que se lleva a cabo en la práctica (Alarcón y Enríquez, 2010; Das y Chatterjee, 2015), generando confusiones y provocando que el término sea utilizado en diferentes órdenes y sectores para referirse a cualquier actividad que se desarrolla en un área natural protegida (Bringas y Ojeda, 2000; Guerrero, 2010).

A pesar de la existencia de literatura que destaca sus beneficios potenciales e historias de éxito (Das y Chatterjee, 2015), existe una cantidad cada vez mayor de investigaciones que ponen en evidencia las preocupantes deficiencias en la materia (Das y Chatterjee, 2015; Ross y Wall, 1999), tanto en la planeación, la gestión, el monitoreo, como en los resultados esperados en los temas económico, social y ambiental. En algunos casos se ha observado que el ecoturismo propicia impactos negativos similares a los causados por el turismo masivo (Bringas y Ojeda, 2000). Es posible que una comunidad llegue a poner por encima el interés económico y dejar de lado u omitir otros pilares como la conservación de la naturaleza y la cultura local (Alarcón y Enríquez, 2010; Conanp, 2018).

De igual manera pueden surgir situaciones no deseadas como la distribución inequitativa de ingresos, la migración y el desplazamiento de tierras (Conanp, 2018; Das y Chatterjee, 2015; Gómez y Dredge, 2003; PNUMA y OMT, 2002; Vargas y Brenner, 2013); también se generan conflictos socio-culturales al existir participación parcial o nula de la población local (Alarcón y Enríquez, 2010; Das y Chatterjee, 2015; Gómez y Dredge, 2003; Obombo y Velarde, 2018; Vargas y Brenner, 2013), además de un debilitamiento de la identidad cultural, la comercialización de tradiciones (Conanp, 2018; PNUMA y OMT, 2002) y un acelerado crecimiento urbano.

Al desarrollarse en espacios naturales se corre el riesgo de alterar y propiciar cambios en el comportamiento de las especies, erosión de suelos, cambios en el paisaje (Gómez y Dredge, 2003; Obombo y Velarde, 2018; Orgaz, 2014; PNUMA y OMT, 2002), contaminación, cambios en los usos de suelos y la cobertura vegetal (Conanp, 2018; Vargas y Brenner, 2013), introducción de especies exóticas (Conanp, 2018), destrucción de hábitats sensibles (Gómez y Dredge, 2003; PNUMA y OMT, 2002) y sobrecarga turística (Alarcón y Enríquez, 2010; Conanp, 2018; Das y Chatterjee, 2015). 


\section{Metodología}

Como se expresó previamente, para la valoración del grado de ajuste de los proyectos ecoturísticos y afines desarrollados en San Cristóbal de las Casas, Chiapas, se usó como guía la Norma 133 que elaboró la Semarnat, en la cual se establecen los requisitos y especificaciones de sustentabilidad del ecoturismo. El enfoque de este trabajo es principalmente cuantitativo ya que el levantamiento de información se realizó considerando la aplicación de tres capítulos que contienen una serie de criterios con valores numéricos asociados, los cuales arrojan un puntaje previamente establecido.

El primer capítulo se denomina Requisitos generales y tiene un valor de 55 puntos que está integrado por 49 criterios en donde se evalúan temas de planeación, directrices de manejo, indicadores de impacto y disposiciones jurídicas. El segundo capítulo que se titula Requisitos de instalaciones, tiene un valor total de 80 puntos y se valoran 35 criterios con aspectos relacionados a la ubicación, el diseño de las instalaciones y la gestión ambiental que contempla el manejo de residuos sólidos urbanos, uso de agua, energía y las acciones de reducción de productos empaquetados y uso de productos ambientalmente responsables.

El tercer y último capítulo que se intitula Actividades y cuenta con un total de 57 puntos, formado por 17 criterios en los que se observan temas de interpretación y educación ambiental, establecimiento de senderos y conservación, así como manejo y aprovechamiento sustentable de la vida silvestre. Para otorgar el puntaje de cada capítulo, la Norma 133 indica que para cada criterio se debe cumplir con un medio de verificación que consta de hacer inspecciones visuales en las áreas y revisión de documentos que deben tener establecidos.

\subsection{Levantamiento de la información}

Debido a que no todos los sitios en el estudio presentan las mismas características y por lo tanto no aplican a varios de los criterios que marca la Norma, se realizó un ajuste de valores tal y como esta propone. Como primer paso se efectuó un recorrido por los cuatro sitios elegidos para identificar los criterios que no aplican. Una vez hecha la primera valoración se hizo el ajuste mencionado, de tal modo que la puntuación total por criterios se mantiene constante y se alcanza con los aspectos que sí corresponden. Por ejemplo, el apartado de ecosistemas acuáticos que está enfocado en evaluar actividades de playa se omitió, ya que la región donde se ubican los proyectos no aplica para este rubro. El ajuste se realizó de la siguiente manera: 
a) Se identificaron los criterios de cada capítulo que no aplican y se les asignó un valor base 0 .

b) Se calculó la suma total de los criterios que sí aplican.

c) Después de realizar los pasos 1 y 2 se utilizó la siguiente fórmula para obtener la nueva puntuación ajustada.

$$
P A=\left(\frac{P B i}{S}\right) * P C
$$

Donde:

$\mathrm{PA}=$ puntuación ajustada;

$\mathrm{PB}_{\mathrm{i}}=$ puntuación base del criterio;

$\mathrm{S}$ = suma de los criterios o subcriterios aplicables para cada sitio (paso 2) y $\mathrm{PC}=$ valor del criterio.

d) Finalmente, se procedió al paso 3 para cada criterio aplicable en los sitios. Los nuevos valores obtenidos se sumaron y el resultado fue el mismo total de puntos establecidos en la Norma 133.

Una vez obtenidas las nuevas puntuaciones por criterios y subcriterios que aplicaban, se hicieron nuevos recorridos para asignar una puntuación que correspondiera a cada sitio. Las notas derivadas se compararon con los valores que establece la Norma para considerar si un sitio tiene un manejo ecoturístico. De igual modo, se realizaron entrevistas con el personal de cada área para valorar los documentos de creación, planeación, directrices de manejo, disposiciones jurídicas y las acciones y medidas para mantener controlada su actividad. Se realizaron caminatas y observaciones para aquellos criterios y subcriterios que correspondían a las inspecciones visuales.

\section{Resultados: aplicación de la Norma 133 en los parques ecoturísticos}

Los resultados se describen a continuación, de acuerdo con los tres capítulos que maneja la Norma 133 en México.

\subsection{Diagnóstico, planeación y manejo de los sitios (capítulo 1)}

La suma de los puntajes alcanzados por sitio fue mayor para Moxviquil (38.76), seguido por San José (37.61), Arcotete (14.57) y finalmente Rancho Nuevo (13.57) (ver tabla 1). Los criterios del capítulo 1 más penalizados fueron la planeación territo- 
rial y tampoco recibió valor la estimación del impacto ambiental de la actividad ecoturística. De los cuatros sitios, el Arcotete y Rancho Nuevo no contaron con un documento de planeación que incluyera el diagnóstico para valorar los recursos naturales con los que cuentan, así como los posibles impactos por el turismo. No hubo al inicio de los proyectos una delimitación de áreas de conservación, restauración y aprovechamiento turístico. Asimismo, las construcciones y actividades no se guiaron por una planeación inicial.

En Arcotete y Rancho Nuevo no se han desarrollado estrategias de educación ambiental con respecto al área natural que alberga su sitio. Moxviquil y Parque San José cuentan con documentos de planeación, en ambos se mantienen listados de especies y han definido zonas para la conservación, restauración y aprovechamiento turístico. También han generado códigos de ética sobre su compromiso en el cuidado del medio ambiente. Ninguno de los cuatro sitios ha definido la capacidad de carga turística, no han realizado estudios de mercado y tampoco han ideado un mecanismo para monitorear periódicamente la actividad que realizan. No obstante, cabe resaltar que los cuatro sitios cuentan con los permisos para desarrollar turismo (ver tabla 1).

Tabla 1. Puntajes de cuatro sitios ecoturísticos de San Cristóbal de las Casas por tema del capítulo 1 de la Norma 133

\begin{tabular}{|c|c|c|c|c|c|}
\hline Temas & Criterios & Arcotete & $\begin{array}{c}\text { Rancho } \\
\text { Nuevo }\end{array}$ & Moxviquil \\
\hline San José
\end{tabular}

Fuente: Elaboración propia. 


\subsection{Instalaciones (capítulo 2)}

Para el capítulo 2 referente a las instalaciones, los sitios con el menor puntaje fueron Arcotete (44.36) y Rancho Nuevo (43.79), mientras que Moxviquil (73.32) tuvo el mayor valor seguido por San José (66.62) (ver tabla 2). Para el tema de ubicación de las instalaciones, los cuatro espacios presentan construcciones que no rebasan las copas de los árboles, los materiales son de madera, adobe, concreto con colores armónicos que se adaptan al paisaje. Entre ellos, Moxviquil es el que ha desarrollado más estrategias de gestión ambiental: han instalado sistemas de captación de agua, baños secos, un filtro de aguas grises, canales de oxidación, vermifiltro, un biodigestor, calentador solar, estufa ahorradora de leña; además, cuentan con área de compostaje, así como un plan de manejo de residuos sólidos urbanos.

El Parque San José tiene un sistema de captación de agua, letreros de ahorro y uso del vital líquido, un área de compostaje y separación de basura. En el sitio de Arcotete solo emplean paneles solares para la luz de la cabaña, restaurante y baños y respecto a la basura solo separan el plástico PET.

Rancho Nuevo es el sitio que no ha implementado una estrategia para reducir el consumo de recursos, únicamente manejan el PET para su reciclaje. Las cuatro áreas han trabajado campañas de reforestación por iniciativa propia y en adhesión a las campañas municipales, que a su vez responden a solicitudes de la federación. Sin embargo, faltan para el Arcotete y Rancho Nuevo listados de especies de sus áreas y programas de protección de vida silvestre.

En los casos de Moxviquil y San José, son espacios que tienen ejemplares de fauna y flora silvestre en confinamiento, pero cuentan con el registro y el permiso como Unidades de Manejo para la Conservación de la Vida Silvestre (UMAs) autorizadas por la Semarnat. En Moxviquil se tiene un programa para proteger el chipe cachetes amarillos (Setophaga chrysoparia Sclater, PL y Salvin) ave que de acuerdo con la Unión internacional para la Conservación de la Naturaleza (UICN) se encuentra en peligro (Biocomuni, s.f). Aunque comentan que todavía faltan acciones para proteger anfibios, reptiles y mamíferos dentro del área, por parte del Parque San José trabajan con la protección del dragoncito de labios rojos (Abronia Lythrochila), salamandras y la nauyaca de frío (Cerrophidion tsotsilorum). Cabe señalar que Moxviquil y San José permiten la investigación. 
El criterio de acciones de compra responsable y uso sustentable de productos amigables con el ambiente se observó únicamente en los sitios Arcotete y Rancho Nuevo, que tienen productos y comidas a la venta. Aunque en la entrevista mencionaron que tienen reglamentos para reducir la venta de productos de lenta degradación, en los recorridos se observó comercialización en la que prevalecían los productos empaquetados.

Tabla 2. Puntajes de cuatro sitios ecoturísticos de San Cristóbal de las Casas por temas del capítulo 2 de la Norma 133

\begin{tabular}{|c|c|c|c|c|c|}
\hline Temas & Criterios & Arcotete & $\begin{array}{l}\text { Rancho } \\
\text { Nuevo }\end{array}$ & Moxviquil & $\begin{array}{l}\text { Parque San } \\
\text { José }\end{array}$ \\
\hline $\begin{array}{l}\text { Ubicación de las } \\
\text { instalaciones (26) }\end{array}$ & $\begin{array}{l}\text { Mapas, características de las instalaciones; } \\
\text { arquitectura y colores amigables con el } \\
\text { ambiente, materiales de construcción, } \\
\text { miradores y torres de observación }\end{array}$ & 17 & 10 & 26 & 26 \\
\hline \multirow{6}{*}{$\begin{array}{c}\text { Gestión ambiental } \\
\text { (54) }\end{array}$} & Uso eficiente del agua & 2 & 3 & 12.38 & 7.52 \\
\hline & Uso eficiente de la energía & 4.86 & 2.29 & 4.5 & 3 \\
\hline & $\begin{array}{l}\text { Acciones de conservación y uso } \\
\text { sustentable de la vida silvestre }\end{array}$ & 12 & 10 & 16.4 & 16.4 \\
\hline & Programa integral de residuos & 7 & 7 & 14.04 & 13.7 \\
\hline & $\begin{array}{l}\text { Acciones de compra y uso de productos } \\
\text { ambientalmente responsables }\end{array}$ & 1.5 & 1.5 & No aplica & No aplica \\
\hline & Total & 27.36 & 23.79 & 47.32 & 40.62 \\
\hline & Totales por tema & 44.36 & 33.79 & 73.32 & 66.62 \\
\hline
\end{tabular}

Fuente: Elaboración propia.

\subsection{Actividades (capítulo 3)}

Para el capítulo Actividades, la Norma 133 maneja un total de 57 puntos distribuidos en tres temas: a) interpretación ambiental (27.2 puntos), b) senderos (14.23) y c) conservación, manejo y aprovechamiento sustentable de la vida silvestre (15.54). Los resultados de cada sitio se ordenan ascendentemente de la siguiente manera: Rancho Nuevo (8.47), Arcotete (25.3), Moxviquil (32.36) y San José (34.95), (ver tabla 3).

Arcotete y Rancho Nuevo no ofrecen ninguna actividad de interpretación y educación como marca la normativa, tampoco han desarrollado manuales con métodos y técnicas para dar a conocer los recursos naturales con los que cuentan. Por otro lado, aunque Arcotete tiene senderos estos no son utilizados para interpretación ambiental. En el caso de Rancho Nuevo, en la entrevista con el presidente en turno 
mencionó que sí cuentan con senderos, pero cuando se realizó la inspección visual las únicas rutas establecidas fueron las de los recorridos a caballo.

En Moxviquil y San José, aunque no cuentan con un manual de interpretación como dispone la Norma 133, han desarrollado diversos mecanismos como: senderos con letreros de información, folletos con datos del lugar, cursos de capacitación para el personal, talleres de educación ambiental de acuerdo con las necesidades y temas de interés de los visitantes y escuelas. Arcotete y Rancho Nuevo no cuentan con guías, mientras que en Moxviquil y San José los recorridos guiados, cuando son solicitados, se llevan a cabo por parte del personal, aunque mencionan que no se encuentran certificados bajo la NOM-09-TUR-2002.

Tabla 3. Puntajes alcanzados por cuatro sitios ecoturísticos en San Cristóbal de las Casas, con base en los criterios capítulo 3 de la Norma 133

\begin{tabular}{|c|c|c|c|c|c|}
\hline Temas & Criterios & Arcotete & $\begin{array}{l}\text { Rancho } \\
\text { Nuevo }\end{array}$ & Moxviquil & $\begin{array}{l}\text { Parque } \\
\text { San José }\end{array}$ \\
\hline $\begin{array}{l}\text { Interpretación } \\
\text { ambiental (27.2) }\end{array}$ & $\begin{array}{l}\text { Estrategias y temas de interpretación, } \\
\text { manuales de interpretación, guías } \\
\text { acreditados bajo la NOM-09-TUR-2002 y } \\
\text { cuestionarios de satisfacción para el turista }\end{array}$ & 0 & 0 & 5.18 & 5.18 \\
\hline Senderos (14.25) & $\begin{array}{l}\text { Diseño de senderos, capacidad de carga, } \\
\text { sistema de señalética diseñada para } \\
\text { orientar durante los recorridos y acciones } \\
\text { de mantenimiento de los senderos }\end{array}$ & 9.76 & 5.88 & 11.64 & 14.64 \\
\hline \multicolumn{2}{|c|}{$\begin{array}{c}\text { Conservación, manejo y aprovechamiento sustentable } \\
\text { de vida silvestre (15.54) }\end{array}$} & 15.54 & 2.59 & 15.54 & 15.54 \\
\hline \multicolumn{2}{|r|}{ Total } & 25.3 & 8.47 & 32.36 & 35.36 \\
\hline
\end{tabular}

Fuente: Elaboración propia.

Durante los recorridos se observó que en Arcotete y Rancho Nuevo se permiten actividades como paseo a caballo, tirolesa y escalada en roca que no corresponden a la modalidad del ecoturismo sino al turismo de aventura (ver tabla 4). 
Tabla 4. Actividades que se permiten en cuatro sitios ecoturísticos en San Cristóbal de las Casas y la modalidad a la que pertenecen

\begin{tabular}{|c|c|c|c|c|c|}
\hline Modalidad & Actividad & Arcotete & $\begin{array}{l}\text { Rancho } \\
\text { Nuevo }\end{array}$ & Moxviquil & $\begin{array}{l}\text { San } \\
\text { José }\end{array}$ \\
\hline \multirow{4}{*}{ Ecoturismo } & Senderismo & $x$ & & $x$ & $x$ \\
\hline & Recorrido por el orquidiario & & & $\mathrm{x}$ & \\
\hline & $\begin{array}{l}\text { Recorrido por el zoológico, jardín del bosque, } \\
\text { epifitario y museos }\end{array}$ & & & & $\mathrm{x}$ \\
\hline & Talleres de educación ambiental & & & $x$ & $x$ \\
\hline \multirow{5}{*}{$\begin{array}{l}\text { Turismo de } \\
\text { aventura }\end{array}$} & Recorrido por la gruta & $\mathrm{x}$ & $\mathrm{x}$ & & \\
\hline & Tirolesas & $\mathrm{x}$ & $\mathrm{x}$ & & \\
\hline & Escalada en roca & $x$ & & & \\
\hline & Paseo por canoas & $\mathrm{x}$ & & & \\
\hline & Paseos a caballo & $\mathrm{x}$ & $\mathrm{x}$ & & \\
\hline
\end{tabular}

Fuente: Elaboración propia con base en Sectur (2004).

\subsection{Comparación de resultados por capítulos}

En general, los sitios alcanzaron puntajes por debajo del límite mínimo, tanto por capítulo como en el total, para considerar que su manejo se ajuste a la sustentabilidad que se define en la Norma 133 (ver tabla 5) con excepción de Moxviquil, que fue el único que cumplió con los requisitos para las instalaciones, entrando dentro del intervalo de un manejo sustentable.

Tabla 5. Puntaje total y por capítulo obtenido por los sitios ecoturísticos de San Cristóbal de las Casas

\begin{tabular}{|c|c|c|c|c|}
\hline Capítulos & Arcotete & $\begin{array}{c}\text { Rancho } \\
\text { Nuevo }\end{array}$ & Moxviquil & $\begin{array}{c}\text { San } \\
\text { José }\end{array}$ \\
\hline 1. Diagnóstico, planeación y manejo de los sitios (45-55 puntos) & 14.57 & 13.57 & 38.76 & 37.61 \\
\hline 2. Requisitos para las instalaciones (70-80 puntos) & 44.36 & 43.79 & 73.32 & 66.62 \\
\hline 3. Requisitos para las actividades (47-57 puntos) & 25.3 & 8.47 & 32.36 & 35.36 \\
\hline Total (162-192) & 84.23 & 65.83 & 144.44 & 139.59 \\
\hline
\end{tabular}

Nota: Entre paréntesis se presenta el puntaje mínimo y máximo que se establece en la Norma 133 para ponderar un manejo sustentable.

Fuente: Elaboración propia. 


\section{Discusión: la aplicación de la normativa}

Los resultados obtenidos muestran que el manejo de los sitios ecoturísticos Arcotete y Rancho Nuevo dista de manera importante de lo establecido en la Norma 133, eso puede deberse en gran medida a la falta de conocimiento de la existencia y/o el contenido de esta normatividad por parte de los operadores de los sitios. En general, el desconocimiento del marco jurídico que regula la modalidad genera prácticas inapropiadas (López y Palomino, 2018).

Como se aprecia en la tabla 5, la Norma 133 utiliza un rango mínimo de 162 puntos y un máximo de 192 para alcanzar la certificación. En los casos de Moxviquil y San José, áreas manejadas por el sector privado la primera y el estatal la última, se observa que fueron las que estuvieron más cerca del mínimo establecido por la Norma para considerarse con un manejo sustentable (18 y 22 puntos respectivamente por debajo del mínimo). Es importante resaltar que en lo referente al capítulo 2, Requisitos para las instalaciones, ambas alcanzaron el puntaje mínimo requerido. En particular se considera que estos sitios pueden mejorar su situación de manera relativamente fácil, poniendo especial atención en aquellos rubros que fueron evaluados por debajo del mínimo.

Con base en los totales obtenidos en cada sitio, los espacios aún no logran un manejo sustentable, tal como se especifica y valora en la Norma 133. Esto puede incluso sugerir una interpretación incorrecta de lo que implica un proyecto de corte sustentable en al menos dos de los sitios que se autodenominan como ecoturísticos, lo que suele suceder cuando los proyectos responden a los intereses económicos o políticos de ciertos actores, pero desafortunadamente tienen una escasa o nula visión para brindar soluciones a problemas ambientales (Manuel y Meza, 2014).

Además, la situación se agrava cuando las organizaciones sociales que operan el sitio desconocen lo que implica administrar un proyecto turístico bajo el paradigma de la sostenibilidad, de modo que la proyección del impacto social y cultural del proyecto se desvía de los objetivos (Bringas y Ojeda, 2000; Manuel y Meza, 2014; Vanegas y Cadavid, 2006). Sin embargo, el presente estudio no incluyó un análisis sobre el conocimiento, actitud y percepción de este tipo de turismo entre los actores involucrados, lo cual podría ayudar a entender los resultados que se encontraron con respecto a los índices de la Norma 133.

En el apartado de diagnóstico, planeación y manejo de los sitios se observa que los proyectos de Arcotete y Rancho Nuevo han sido poco planificados en términos de 
uso del espacio y en los diagnósticos sociales, económicos y ambientales que permiten tener un conocimiento general del territorio, lo cual ayuda a planear y crear instrumentos que posibiliten en este caso prevenir daños en el ambiente por las actividades realizadas (Semarnat, 2006).

Según Obombo y Velarde (2018) la creación de instrumentos de conservación en el ecoturismo será determinada por factores como la situación económica. La falta de planeación en los sitios Arcotete y Rancho Nuevo se debe posiblemente a que se identificó el atractivo focal como una oportunidad de generar un ingreso extra (Obombo y Velarde, 2018), sin tener en cuenta las características generales del espacio, dando como resultado que utilicen el término "ecoturístico" solo porque están en un área natural (Bringas y Ojeda, 2000; Guerrero, 2010).

Igualmente, se detectó que se promueven actividades que no corresponden propiamente con la práctica del ecoturismo (Björk, 2000; Ross y Wall, 1999), lo que refuerza la idea de una falta de conocimiento de la Norma 133, así como de la filosofía de sustentabilidad inherente. La falta de o poca planeación y monitoreo de estos espacios hace que no consideren el ordenamiento ecológico, ni se establezca la capacidad de carga o se realicen estudios de viabilidad (Gómez y Dredge, 2003).

Por su parte, los sitios San José y Moxviquil, al ser espacios destinados para la conservación, cuentan con planes de manejo en los que se definen las áreas de preservación, restauración y de uso turístico. En este sentido se observa una mayor planeación en términos de un ordenamiento del territorio. Sin embargo, ambos sitios carecen de una planeación en torno a la capacidad de carga, protocolos de control de visitantes, así como los estudios de mercado para definir el perfil del turista.

Lo anterior se debe en parte a que la ciudad de San Cristóbal de las Casas, cercana a estos sitios, se ha caracterizado por atraer a un turismo de corte cultural, identificado por la Sectur (2004) como parte del turismo convencional o masivo que, como es sabido, centra su atención en el gran volumen de flujos turísticos con el fin de generar una mayor cantidad de recursos económicos, soslayando con frecuencia las dimensiones sociales y ambientales (Semarnat, 2017). Por su parte, Moxviquil y San José han establecido formas para orientar el comportamiento de turistas y visitantes locales dentro de sus espacios, así como códigos de ética dirigidos al personal con relación al cuidado del medio ambiente, la comunidad y los turistas, lo cual se apega a los principios establecidos por el ecoturismo (PNUMA y OMT, 2002; TIES, 2015). 
Rancho Nuevo y Arcotete carecen de códigos y estrategias dirigidas a la conservación de sus elementos naturales. Manuel y Meza (2014) plantean que en el caso del ecoturismo, al tratarse de un concepto polisémico, se corre el riesgo de atraer actores que solo buscan generar recursos económicos y se puede caer en prácticas del turismo masivo o convencional. Esto implica una exclusión de la conservación y de la sociedad, aunque se llevan a cabo acciones vinculadas con estos aspectos, realmente existe una priorización de lo económico que se traduce en una mayor presión sobre el ambiente (Conanp, 2018; Vargas y Brenner, 2013).

Aunque no existe una sola metodología para evaluar el desempeño de los espacios o proyectos estudiados, debido en parte a que cada región tiene sus propias características económicas, biológicas y culturales (Lauterio-Martínez et al., 2018), sí es importante la creación de indicadores que permitan una valoración anual o bianual de las actividades ecoturísticas y el cumplimiento de los objetivos que se les asocian, en ese sentido las certificaciones toman relevancia (Holub, 2015). Por lo tanto, si hubiera desacuerdo con la puntuación alcanzada con base en la Norma 133, la alternativa es que se generen los respectivos indicadores con pertinencia y objetividad, para mostrar tanto al gremio de socios de los proyectos como a las autoridades ambientales nacionales, a los visitantes y a la sociedad en general la eficiencia del manejo sustentable de los proyectos.

La Norma 133 establece que las únicas actividades ecoturísticas que se pueden desarrollar en los sitios son de educación ambiental e interpretación, así como otras no previstas pero que carezcan de impacto sobre el escenario natural del sitio. Sin embargo, en el Arcotete y Rancho Nuevo se detectaron servicios que corresponden al turismo de aventura, como son el paseo a caballo, en canoa, la tirolesa y escalada en roca (Semarnat, 2017).

En ambos casos, es posible que el término en cuestión se confunda con la modalidad de turismo de aventura porque tanto en Rancho Nuevo como en Arcotete desarrollan actividades que tienen que ver con esta última modalidad, establecidas en la Norma NOM-09-TUR-2002 de guías especializados en actividades específicas (Sectur, 2003) y la NOM-011-TUR-2001 sobre las empresas de turismo de aventura (Sectur, 2002).

Bringas y Ojeda (2000) y Guerrero (2010) señalan que el hecho de crear un espacio turístico en un área natural y desarrollar actividades al aire libre no significa que se hace ecoturismo. La confusión que conduce al éxito o al fracaso de este tipo de 
turismo, depende del contexto y la cultura local en el que se desarrolle. (Obombo y Velarde, 2018). De igual forma, los intereses de las personas involucradas pueden llegar a enfocarse en prácticas que no concuerdan con lo que establece esta forma de turismo, considerada de bajo impacto (Manuel y Meza, 2014).

Otro aspecto que llama la atención es que en Arcotete y Rancho Nuevo no se observó algún programa de educación ambiental con estrategias y temas que permitan dar a conocer los elementos naturales que albergan los espacios, que es un punto fundamental en términos de la Norma. Piñar et al. (2012) demuestran en su estudio que existe la necesidad de crear espacios de aprendizaje donde la educación ambiental sea el punto focal, para que los actores involucrados puedan trabajar en conjunto a fin de lograr estrategias que ayuden a alcanzar prácticas más sustentables.

En el mismo tenor, Björk (2000) enfatiza que la protagonista en todo esto es la naturaleza y, por tanto, esta debe verse en un contexto más amplio, en donde existan áreas genuinas, la posibilidad de participar, estar activo y aprender, esto quiere decir que el turista y el prestador de servicios generen experiencias únicas. En ese sentido, a Rancho Nuevo y Arcotete les falta trabajo y capacitación para conocer y valorar todo el capital natural que estos sitios albergan.

Bringas y Ojeda (2000) señalan que el desarrollo de este tipo de turismo requiere una inversión en capacitación más que en infraestructura, ya que la atención de los nuevos segmentos de mercado demanda una alta preparación del personal que ofrece el servicio, que en este caso es gente local, con el fin de generar experiencias únicas y recomendables (Conanp, 2018).

En Moxviquil y San José se observa otra dinámica: aunque propiamente su giro no es el ecoturismo, en el capítulo de las actividades obtuvieron las puntuaciones más altas, puesto que dentro de estos espacios las acciones que realizan tienen como componente principal la educación ambiental. Al ser espacios destinados a la conservación y recibir constantes capacitaciones, ambos sitios procuran que sus visitantes conozcan la naturaleza que los rodea y que valoren la importancia de cuidarla; asimismo, promueven la investigación en sus espacios y esto permite que suceda lo que mencionan Ross y Wall (1999): se convierten en laboratorios, museos vivos, retiros, refugios y escuelas al aire libre, que permiten brindar oportunidades únicas e interactivas tanto para el visitante como para la población local. En estos casos se observa que se cumplen los principios en los que se fundamenta el ecoturismo (PNUMA y OMT, 2002; TIES, 2015). 
Un aspecto importante de la certificación bajo la Norma 133, es que permite distinguir a los prestadores de servicios que realizan actividades sostenibles (Cancino, 2016); además, mediante la evaluación se logran identificar las fortalezas y debilidades de un sitio. Dicha normativa, al hacer una evaluación de la planeación, forma de manejo y acciones que se implementan en un proyecto ecoturístico, estimula la búsqueda por parte de los prestadores de servicios de estrategias (bajo sus propios indicadores) que permitan controlar, monitorear y brindar un servicio de calidad (Camacho-Ruiz et al., 2016) para así cumplir con la conservación, el bienestar de la sociedad local y el desarrollo económico.

\section{Conclusiones}

Desde la perspectiva y métrica de la Norma 133 y teniendo presentes los principios de la sostenibilidad en los que se basa el ecoturismo, es posible afirmar que todavía ambos aspectos son incipientes en las zonas de estudio, principalmente en las áreas que son controladas por las comunidades, en las cuales se aprecia una falta de indicadores locales para monitorear los impactos y mantener regulada la actividad.

Igualmente, se percibe un insuficiente conocimiento de las implicaciones de un desarrollo ecoturístico, así como de los lineamientos y las certificaciones por parte de los operadores de estos sitios, en los cuales sería recomendable optar por la certificación de la Norma 133, ya que, tal como lo plantea Cancino (2016), una forma de lograr una adecuada gestión de los proyectos ecoturísticos es por medio de las certificaciones y normas.

Otro punto importante, es que existe una confusión acerca de las actividades que se establecen dentro del turismo de bajo impacto; resulta necesario profundizar para reconocer las causas de las prácticas locales que orienta dicha actividad en los espacios. Los resultados obtenidos coinciden con los encontrados por López y Palomino $(2008 ; 2018)$, quienes proponen que los desarrollos del ecoturismo en México se construyen desde la concepción del tipo convencional, dejando a un lado la conservación y la creación de códigos de conducta, centrándose principalmente en el desarrollo de planta turística.

De la misma manera, es necesario que la Norma oficial 133 precise algunos criterios y que sea más explícita en sus requisitos; además, requiere agregar otros capítulos en los que evalúen la participación de la comunidad y la parte económica, para re- 
forzar y obtener un trabajo completo, pues este tipo turismo, que se enmarca en la sostenibilidad, busca el equilibrio entre lo económico, social y ambiental.

Camacho-Ruiz et al. (2016) plantean que la Norma 133 podría ser un buen método de evaluación eficaz si en ella se establecieran parámetros que ayudaran a medir y a monitorear periódicamente las actividades y el desarrollo de un proyecto, con el fin de identificar los posibles impactos, ya que adaptar el espacio con los criterios, documentos y obtener la información para la certificación no son garantía para transitar hacia la sostenibilidad, si no se le da un seguimiento y evaluación constante.

En este orden de ideas y en busca de que las zonas de estudio alcancen los estándares contemplados en la Norma 133, sería recomendable que en los cuatro sitios estudiados se estime la capacidad de carga y se realicen estudios de mercado, con el fin de identificar el segmento de ecoturistas. Particularmente en los casos de Arcotete y Rancho Nuevo, se sugiere implementar programas de capacitación en atención a clientes, manejo de grupo y en temas de cuidado y protección de la naturaleza; realizar un inventario de recursos naturales (listados de especies) y establecer senderos educativos, así como certificar a guías locales para recorridos de educación e interpretación ambiental.

\section{Referencias}

Alarcón, P. y Enríquez, P. L. (2010). Implicaciones y contradicciones del ecoturismo en La Sima de las Cotorras, Ocozocoautla de Espinoza, Chiapas [tesis de maestría, El Colegio de la Frontera Sur]. Biblioteca SIBE. https://bit.ly/2Us9kmb

Biocomuni. (s.f.). Chipe cachetes amarillos. https://bit.ly/3wn9qcf

Björk, P. (2000). Ecotourism from a conceptual perspective, an extended definition of a unique tourism form. International Journal of Tourism Research, 2(3), 189-202. https://doi.org/10.1002/(SICI)1522-1970(200005/06)2:3<189::AIDJTR195>3.0.CO;2-T

Bringas, N. L. y Ojeda, L. (2000). El ecoturismo: ¿una nueva modalidad del turismo de masas? Economía, Sociedad y Territorio, 2(7), 373-403. https://doi.org/ 10.22136/est002000436 
Camacho-Ruiz, E., Carrillo-Reyes, A., Rioja-Paradela, T. M. y Espinoza-Medinilla, E. E. (2016). Indicadores de sostenibilidad para el ecoturismo en México: estado actual. LiminaR, 14(1), 156-168. https://bit.ly/3wq3lvk

Cancino, J. (2016). Programas de certificación internacional para PYMES de turismo alternativo en México. Turismo y Desarrollo Local, 9(21), 1-11. https://bit.ly/ $2 \mathrm{TKps} 2 \mathrm{r}$

Chanona-Gómez, F., Álvarez Gutiérrez, P. E. y Pérez-Luna, Y. (2019). Macromycetes of the San José educational park, municipality of Zinacantan, Chiapas, Mexico. Acta Universitaria, 29, 1-13. https://doi.org/10.15174/au.2019.2127

Comisión Nacional de Áreas Naturales Protegidas (Conanp). (2018). Marcoestratégico de turismo sustentable en áreas naturales protegidas de México. https://bit.ly/ 3hphQeA

Das, M. y Chatterjee, B. (2015). Ecotourism: a panacea or a predicament? Tourism Management Perspectives, 14, 3-16. https://doi.org/10.1016/j.tmp.2015.01.002

Gómez, O. y Dredge, D. (2003). Hacia un turismo sustentable. Biodiversitas, 51, 8-11. Guerrero, R. (2010). Ecoturismo mexicano la promesa, la realidad y el futuro. Un análisis situacional mediante estudios de caso. El Periplo Sustentable, 18, 37-67. https://bit.ly/3ABHkx8

Henríquez, E. (2012). Las Grutas de Rancho Nuevo cautivan la imaginación con figuras caprichosas. La Jornada. https://bit.ly/3hLYwHw

Holub, M. (2015). Ecotourism certification programs: standards and benefits. Baltic Journal of Economic Studies, 1, 67-74. https://doi.org/10.30525/ 2256-0742/2015-1-1-67-74

Honey, M. y Rome, A. (2001). Protecting paradise: certification programs for sustainable tourism and ecotourism. Institute for Policy Studies. https://bit.ly/3hJKENR

Instituto Nacional de Estadística y Geografía (INEGI). (2017). Anuario estadístico y geográfico de Chiapas 2017. https://bit.ly/3ACF3lq

Lauterio-Martínez, C. L., Hernández-Trejo, V. Á., Ortega-Rubio, A., Olmos-Martínez, E., Ibáñez-Pérez, R. M. y Bobadilla-Jiménez, M. (2018). The value of ecotourism and ecosystem services in Espiritu Santo Island National Park, Mexico. En A. Ortega-Rubio (Ed.), Mexican natural resources management and biodiversity conservation (pp. 431-453). Springer, Cham. https://doi.org/10.1007/ 978-3-319-90584-6_19 
López, G. y Palomino, B. (2008). Políticas públicas y ecoturismo en comunidades indígenas de México. Teoría y Praxis, 4(5), 33-50. https://doi.org/10.22403/ UQROOMX/TYP05/03

López, G. y Palomino, B. (2018). Turismo de naturaleza en comunidades indígenas en México. Universidad Nacional Autónoma de México e Instituto de investigaciones ecnómicas.

Luna, G. (2018). Parque Educativo Bocomtenelté, un espacio abierto al turismo. Diario Ultimátum. https://bit.ly/3jRYGjb

Manuel, M. y Meza, M. (2014). Análisis del ecoturismo como alternativa de desarrollo (México, Costa Rica y Ecuador) [tesina de maestría, El Colegio de la Frontera Sur, Université de Sherbrooke].

Montoya, G. y Hernández, J. (2013). Proyectos de ecoturismo en San Cristóbal de las Casas. En G. Zárate, O. Gordillo y M. Villafuerte (Eds.), Cambios, rupturas y continuidades en la dinámica territorial de San Cristóbal de las Casas, Chiapas (pp. 79-99). Universidad Autónoma de Chiapas.

Moreno, A. (2014). Actores sociales involucrados en la conservación de la biodiversidad: el caso de la reserva ecológica Moxviquil, Chiapas, México [tesis de maestría, El Colegio de la Frontera Sur]. Biblioteca SIBE. https://bit.ly/3dTLhDt

Obombo, K. y Velarde, M. (2018). Ecoturismo y conservación: perspectivas y prácticas en las reservas de la biósfera de Los Tuxtlas, México y Maasai Mara, Kenia. Dimensiones Turísticas, 2(2), 53-77. https://doi.org/10.47557/PMQQ9256

Orgaz, F. (2014). Los impactos económicos, sociales y medioambientales negativos en el ecoturismo: una revisión de la literatura. Nómadas, Critical Journal of Social and Juridical Sciences, 42(2), 139-148. https://doi.org/10.5209/ rev_NOMA.2014.v42.n2.48781

Picado, M., Montoya, G. y Hernández, J. (2014). Ecoturismo y transformaciones socieconómicas y territoriales en el ejido "Río Arcotete", del municipio de San Cristóbal de las Casas, Chiapas. En J. Barragán y M. Maldonado (Eds.), El turismo y desarrollo comunitario. investigaciones y propuestas (pp. 369-393). Colección Academia, Universitaria.

Piñar, Á., García, M. y García, H. (2012). Ecoturismo y educación ambiental para la sustentabilidad en la reserva de la biosfera de Los Tuxtlas (México). Revista de Investigación en Turismo y Desarrollo Local, 5(12). https://bit.ly/2SRrJbC 
Programa de las Naciones Unidas para el Medio Ambiente (PNUMA) y Organización Mundial del Turismo (OMT). (2002, 19-22 de mayo). Declaración de Quebec sobre el ecoturismo. Cumbre Mundial del Ecoturismo. Dirigida por la Organización Mundial del Turismo, Quebec, Canadá. https://bit.ly/36kFTVO

Romero-Águila, E. (2012). Monitoreo y manejo de senderos en la Reserva Ecológica Moxviquil, Chiapas [tesis de maestría, El Colegio de la Frontera Sur]. Biblioteca SIBE. https://bit.ly/3jU5nkW

Ross, S. y Wall, G. (1999). Ecotourism: towards congruence between theory and practice. Tourism Management, 20(1), 123-132. https://doi.org/10.1016/ S0261-5177(98)00098-3

Secretaría de Turismo (Sectur). (2002). Norma Oficial Mexicana NOM-011-TUR-2001, Requisitos de seguridad, información y operación que deben cumplir los prestadores de servicios turísticos de Turismo de Aventura. https://bit.ly/3hnheWS

Secretaría de Turismo (Sectur). (2003). Norma Oficial Mexicana NOM-09-TUR-2002, Que establece los elementos a que deben sujetarse los guías especializados en actividades específicas. https://bit.ly/3jTefHI

Secretaría de Turismo (Sectur). (2004). Turismo alternativo una nueva forma de hacer turismo, Fascículo 1. https://bit.ly/2V8Rmp4

Secretaría de Medio Ambiente y Recursos Naturales (Semarnat). (2006). Introducción al ecoturismo comunitario. https://bit.ly/3hltDUj

Secretaría de Medio Ambiente y Recursos Naturales (Semarnat). (2017). Turismo Sustentable en México. https://bit.ly/3xpwWqg

Secretaría de Medio Ambiente y Recursos Naturales (Semarnat). (2018). NMX-133 ecoturismo sustentable. https://bit.ly/3jZZq5Q

The International Ecotourism Society (TIES). (2015). What is the ecotourism? Definition and principles. https://bit.ly/36yqQYZ

Vanegas, G. y Cadavid, C. (2006). Ecoturismo: instrumento de desarrollo sostenible [monografía, Universidad de Antioquía]. Biblioteca Digital de la Universidad de Antioquía. https://bit.ly/3woPFRI

Vargas, D. y Brenner, L. (2013). Ecoturismo comunitario y conservación ambiental: la experiencia de La Ventanilla, Oaxaca, México. Estudios Sociales, Revista de Alimentación Contemporánea y Desarrollo Regional, 21(41), 31-63. http://dx.doi.org/10.24836/es.v21i41.73 